INPLASY

PROTOCOL

To cite: Xie et al. Effectiveness and safety of Neostigmine injections given at the Zusanli acupoint for treating abdominal distension caused by severe acute pancreatitis(SAP) A protocol for a systematic review and meta-analysis. Inplasy protocol 202170043. doi: 10.37766/inplasy2021.7.0043

Received: 14 July 2021

Published: 14 July 2021

Corresponding author: Xie Yong

xieyong1933@126.com

Author Affiliation: WestChina Hospital, Sichuan University.

Support: NO.2018SZ0390.

Review Stage at time of this submission: The review has not yet started.

Conflicts of interest:

None declared.

\section{Effectiveness and safety of Neostigmine injections given at the Zusanli acupoint for treating abdominal distension caused by severe acute pancreatitis(SAP) A protocol for a systematic review and meta-analysis}

Xie, Y1; Cheng, T2; Liu, BF³ Yu, HF4.

Review question / Objective: P: Patients with abdominal distension caused by severe acute pancreatitis (SAP): Treat with Neostigmine injections given at the Zusanli acupoint; C: Treat without Neostigmine injections given at the Zusanli acupoint; O: Hospital mortality and duration of hospital stays; S: RCTs.

Condition being studied: Neostigmine injections given at the Zusanli acupoint is used to treat for abdominal distension caused by severe acute pancreatitis in China. But at present, there is no systematic evaluation report on its therapeutic effectiveness and safety. This protocol aims to reveal the efficacy and safety of Neostigmine injections given at the Zusanli acupoint for treating abdominal distension caused by severe acute pancreatitis.

INPLASY registration number: This protocol was registered with the International Platform of Registered Systematic Review and Meta-Analysis Protocols (INPLASY) on 14 July 2021 and was last updated on 14 July 2021 (registration number INPLASY202170043).

\section{INTRODUCTION}

Review question / Objective: P: Patients with abdominal distension caused by severe acute pancreatitis (SAP): Treat with Neostigmine injections given at the Zusanli acupoint; C: Treat without Neostigmine injections given at the Zusanli acupoint; 0 : Hospital mortality and duration of hospital stays; S: RCTs.

Condition being studied: Neostigmine injections given at the Zusanli acupoint is used to treat for abdominal distension 
caused by severe acute pancreatitis in China. But at present, there is no systematic evaluation report on its therapeutic effectiveness and safety. This protocol aims to reveal the efficacy and safety of Neostigmine injections given at the Zusanli acupoint for treating abdominal distension caused by severe acute pancreatitis.

\section{METHODS}

Participant or population: Patient age $\geq 18 y$ ears old, a minimum hospital stays of 24h and a diagnosis abdominal distension caused by severe acute pancreatitis.

Intervention: Treat with Neostigmine injections given at the Zusanli acupoint.

Comparator: Treat without Neostigmine injections given at the Zusanli acupoint.

\section{Study designs to be included: RCT.}

Eligibility criteria: The inclusion criteria for the study will include:(1) studies with patient age 18 years old,a minimum hospital stay of $24 \mathrm{~h}$ and a diagnosis of SAP;(2) conference abstracts were only included when they provided adequate relevant information for assessment;(3) the patients with SAP was divided into two groups(treated with Treat with Neostigmine injections given at the Zusanli acupoint or without treat with neostigmine injections given at the Zusanli acupoint); Exclusion criteria will include: age $<18$ years old, patients with mild acute pancreatitis or chronic pancreatitis or pancreas carcinoma or patients with incomplete data.

Information sources: We will search the EMBASE, Wanfang, Web of Knowledge,

$Z \mathbf{h}$ i w a n g, P u b $M$ e d, $\mathbf{W}$ e i $\mathbf{p} u$. ClinicalTrials.gov and Cochrane Library from inception to June 30,2021 to retrieve relevant studies. We will also search citations of relevant primary and review. Authors of abstract in the meeting will be further searched in PubMed for potential full articles. To minimize the risk of publication bias, we will conduct a comprehensive search that included strategies to find published and unpublished studies.

Main outcome(s): Hospital mortality.

Quality assessment / Risk of bias analysis: Risk of bias analysis assessment will be carried out according to the NewcastleOttawa Scale (NOS) to rate the internal validity of the individual studies, and funnel plots will be constructed to assess the risk of publication bias.

Strategy of data synthesis: All pairwise meta-analytic calculations will be performed with Review Manager software(RevMan)version5.3 (Cochrane Collaboration). Heterogeneity will be examined by computing the $Q$ statistic and 12 statistic, and presence of reporting bias by visual inspection of funnel plots. Statistical significance was considered when the $P$ value $<0.05$.

Subgroup analysis: Abdominal distension caused by severe acute pancreatitis patients treated with Neostigmine injections given at the Zusanli acupoint and abdominal distension caused by severe acute pancreatitis patients treated without Neostigmine injections given at the Zusanli acupoint.

Sensitivity analysis: Heterogeneity will be examined by computing the $Q$ statistic and2 statistic, and presence of reporting bias by visual inspection of funnel plots. Statistical significance was considered when the $P$ value $<0.05$.

Country(ies) involved: China.

Keywords: severe acute pancreatitis, Neostigmine, the Zusanli acupoint, duration of hospital stays, meta-analysis, mortality, prognosis, systematic review, traditional Chinese medicine.

Contributions of each author:

Author 1 - XIE YONG.

Email: xieyong1933@126.com

Author 2 - CHENG TAO. 
Email: hxjz_CT1989@163.com

Author 3 - LIU BOFU.

Email: 837718077@qq.com

Author 4 - YU HAIFANG.

Email: yuhaifang@wchscu.cn 